La Revue

des Droits

de l'Homme

\section{La Revue des droits de l'homme}

Revue du Centre de recherches et d'études sur les droits fondamentaux

16 | 2019

Revue des droits de l'homme - $\mathrm{N}^{\circ} 16$

\title{
La race de la violence de genre : analyse de la loi brésilienne contre la violence domestique
}

\section{Márcia Nina Bernardes}

\section{OpenEdition \\ Journals}

Édition électronique

URL : http://journals.openedition.org/revdh/7078

DOI : $10.4000 /$ revdh.7078

ISSN : 2264-119X

\section{Éditeur}

Centre de recherches et d'études sur les droits fondamentaux

\section{Référence électronique}

Márcia Nina Bernardes, «La race de la violence de genre : analyse de la loi brésilienne contre la violence domestique », La Revue des droits de l'homme [En ligne], 16 | 2019, mis en ligne le 02 juillet 2019, consulté le 09 juillet 2020. URL : http://journals.openedition.org/revdh/7078 ; DOI : https:// doi.org/10.4000/revdh.7078

Ce document a été généré automatiquement le 9 juillet 2020

Tous droits réservés 


\title{
La race de la violence de genre : analyse de la loi brésilienne contre la violence domestique
}

\author{
Márcia Nina Bernardes
}

En dépit de la Loi Maria da Penha, les femmes noires sont encore assassinées sans la protection

de l'Etat et sans la protection du mouvement

féministe. Où nous sommes-nous trompées?

Comment est-il possible qu'au cours des 10

dernières années le nombre de femmes noires assassinées ait augmenté de $54 \%$ ? (...) Regardez

votre travail, regardez votre organisation, regardez votre lutte ! Comment est-ce possible?

Jurema Werneck ${ }^{1}$

\section{Introduction}

1 Après une lutte acharnée de plus de 30 ans menée par le mouvement féministe, le Brésil a promulgué la loi 11.340 (Loi Maria da Penha, dorénavant “LMP”) le 7 août 2006 qui combat le phénomène de la violence domestique contre les femmes. La loi est le résultat d'une lutte historique. Elle a aussi un caractère interdisciplinaire car elle ne traite pas seulement de répression, mais aussi de prévention des crimes de violence domestique. Pour autant, les chiffres de la «Mapa da Violencia 2015 », publication annuelle recensant des données quantitatives sur la violence au Brésil, sont décevants. L'édition 2015 de la Mapa montre que si la violence contre les femmes blanches a diminué depuis 2003, celle pratiquée à l'encontre des femmes noires s'est accrue. Entre 2003 et 2013, les meurtres de femmes blanches ont diminué de 9,8\% alors que pour les femmes noires ils ont augmenté $54 \%$. La promulgation de la LMP, en 2006, n'a pas modifié ces chiffres de façon significative. ${ }^{2}$ Si l'on prend comme point de départ la date d'entrée en vigueur de la loi, le nombre de victimes blanches a baissé de 2,1 \% et celui 
de victimes noires a augmenté de $35 \%$. D'après la même étude, en 2013, le taux d'homicides sur les femmes noires était de $66 \%$ plus élevé que celui concernant les femmes blanches. Ces femmes meurent principalement de violences dans le cadre domestique, mais pas exclusivement. ${ }^{3}$

Dans cet article, nous analyserons les effets du racisme structurel sur la construction sociale des hiérarchies sexo-spécifiques afin de distinguer certaines dynamiques importantes du phénomène de la violence contre les femmes. Sans négliger la gravité du racisme, nous - théoriciennes féministes -, agissons souvent comme s'il était possible de le mettre entre parenthèses et de le suspendre le temps de réfléchir sur la violence de genre contre les femmes. Thula Pires, lectrice d'Ochy Curiel, affirme qu'il revient aux intellectuels et aux militants de nommer les catégories qui nourrissent leurs réflexions et leurs stratégies, et surtout, de nommer ce qui a été laissé de côté :

«Une grande partie du mythe de l'universalité et de la neutralité construit par la pensée eurocentriste hégémonique a justement subsisté grâce à la non-désignation de certaines catégories; soit pour les perpétuer comme représentatives de l'universel, soit pour garantir leur rejet $»^{4}$.

3 Au moins depuis les travaux de Simone de Beauvoir, le féminisme hégémonique dénonce la figure du sujet universel comme étant excluant. Ce sujet abstrait, sans corps, serait celui des hommes servant d'archétype à la formulation des institutions publiques. Les femmes dans les sociétés patriarcales sont envisagées comme «l'autre ", comme des objets, à partir de leur corps qui, cependant, ne leur appartient pas : il sert au regard des hommes 5 .

4 Les féministes noires accusent la "blanchitude » d'avoir usé du même artifice comme arme de pouvoir: seuls les corps noirs sont marqués, ont une race; et ils sont subordonnés au sujet blanc. Dans cette dynamique raciale de pouvoir, la catégorie «femmes » fonctionne comme substitut du sujet universel, permettant que les femmes marquées racialement soient réifiées et subalternisées dans les luttes du féminisme hégémonique :

"L'individu abstrait est de l'ordre de la blanchitude, comme une racialité non nommée. Pour des sujets non-blancs, comme le dit Fanon pour parler du schéma corporel, historico-racial, du sujet noir, la subjectivité est déplacée par des regards étrangers et, de ce fait, non reconnue dans ses propres termes. Autrement dit, par le biais de ces processus d'aliénation il devient objet dans un système de suprématie blanche $»^{6}$.

Dans cet article, nous nous intéressons aux perspectives épistémologiques qui affectent la formulation et l'application des politiques publiques, tout en respectant les recommandations de Gayatri Spivack contre «l'habitus" de intellectuels qui ont la prétention de refléter directement la position de ceux qu'ils pensent représenter, comme si eux-mêmes étaient tout à fait transparents. Nous ne prétendons pas faire parler dans cet article la femme noire subalternisée; nous nous attachons à réfléchir sur les «conditions de production de la subalternité » qui empêchent certains corps d'être entendus et d'occuper des positions paritaires dans des sociétés dites démocratiques ${ }^{7}$. À quel moment les femmes noires ont-elles été instrumentalisées et réduites au silence dans le contexte de la LMP ? Notre perspective se rapproche de ce que, dans les "études sur la blanchitude", on appelle l'«alphabétisation raciale » (racial literacy). Nous assumons donc le principe de la blanchitude en tant que position socialement construite qui garantit des privilèges à des corps blancs, l'appropriation 
d'une grammaire et d'un vocabulaire racial et l'interprétation des codes et des pratiques racialisées ${ }^{8}$.

Dans le débat sur l'inexorable imbrication entre race et genre, nous nous pencherons plus particulièrement sur deux questions majeures: (i) dans quelle mesure les catégories «genre » et «femme " sont-elles utiles à l'analyse de la subalternisation si elles ne sont pas travaillées en amont à partir du racisme et d'autres axes d'oppression structurelle, et (ii) dans quelle mesure les femmes noires ont-elles été rendues invisibles dans le processus de mise en œuvre de la LMP ? Nous prenons comme point de départ certains insights des féminismes noirs et des féminismes décoloniaux et postcoloniaux pour penser la manière dont sont produites la précarisation des corps et la répartition injuste de l'exposition à la violence, ainsi que le rôle que joue le droit dans cette dynamique. À l'instar d'Ana Flauzina, notre intention est de questionner, plutôt que condamner, les stratégies du mouvement féministe hégémonique brésilien dans la formulation et le suivi de la LMP9 .

Notre réflexion se veut ici exclusivement théorique et nous n'emploierons pas une méthodologie qualitative. Nous ferons cependant souvent recours à des travaux empiriques menés par différents auteurs et par nous-même à d'autres occasions.

Avant l'analyse proprement dite, il convient de fournir quelques informations sur les nouveautés apportées par la LMP, sur le sens qu'elle prenait pour le mouvement féministe à l'époque de son adoption, sur les modifications apportées par son interprétation jurisprudentielle et sur les acquis et les défauts de son application (1) ${ }^{10}$. Nous rentrerons ensuite dans le débat relatif au racisme structurel du patriarcat et sur la nécessité qu'il y a à reconnaître la dimension raciale des expériences des victimes de la violence de genre. Pour ce faire, nous ferons recours à l'étude de Bruna Jaquetto Pereira qui a interrogé des femmes noires victimes de violence pour comprendre comment ce qu'elle appelle "attentes de genre » sociales divergent de l'expérience concrète de ces femmes. Les idées de Maria Lugones, Lelia Gonzales et Sueli Carneiro sont importantes dans cette discussion (2). Notre réflexion se tournera ensuite vers les politiques publiques axées sur l'élimination de la violence de genre qui, tout comme la LMP, sont réputées être neutres du point de vue de la perspective raciale. Le concept d'intersectionnalité forgé par Kimberle Crenshaw s'avère ici important et permet de mettre en lumière une partie du problème, notamment via les notions de sur-inclusion et de sous-inclusion (3).

9 Une des questions ici soulevées porte sur le cadrage de la discussion de la violence de genre au Brésil qui, grosso modo, est devenue partie intégrante de la discussion sur la violence domestique. Nous reviendrons sur les données issues d'une étude que nous avons conduite entre 2014 et 2015 avec Adriana Vidal et Oliveira et Rodrigo de Souza Costa, ainsi qu'avec divers chercheurs du Master et du programme post-gradué de l'Université Pontificale Catholique de Rio de Janeiro (PUC-Rio) et de l'Université Fédérale Fluminense (UFF) (étude ici nommée «étude élargie »). Dans ce cadre, nous avons analysé 355 procès-verbaux de mesures de protection d'urgence (MPU) délivrés par trois cours spécialement compétentes en matière de violence domestique et familiale contre les femmes dans l'état de Rio de Janeiro. Bien que ce corpus ait été initialement constitué dans le cadre d'un autre projet de recherche, il a permis le développement des interrogations et hypothèses formulées dans le présent article ${ }^{11}$. Nous emploierons également le critère que Mariana Imbelloni Braga Albuquerque et nous-même avions utilisé à partir de l'ensemble de données recueillies dans l'étude 
élargie. Dans un article ultérieur, nous avons aussi sélectionné les MPU concernant les femmes noires et mulâtresses (187 procédures). A partir des récits inclus dans les procès-verbaux, nous avons identifié plusieurs facteurs récurrents qui déclenchent les épisodes de violence présentés à la justice et qui ne sont visibles que si l'on chausse les bonnes lunettes, celles qui permettent de voir les intersections entre race, classe et genre $^{12}$.

\section{Brève présentation et évaluation de la loi 11340/06 (Loi Maria da Penha)}

10 La LMP est le fruit d'une lutte historique des mouvements féministes et de l'effort collectif d'organismes publics brésiliens et internationaux. Elle est devenue une référence internationale sur les questions de violence faite aux femmes ${ }^{13}$. C'est la consécration d'une bataille ardue, de 30 ans de militantisme, d'expériences et de connaissances accumulées sur la problématique de la violence domestique, une des expressions les plus graves d'un ordre social profondément sexiste et injuste qui dégrade et tue les femmes ${ }^{14}$. Le texte de la loi reflète la reconnaissance du fait que la violence domestique est un problème d'ordre public, assorti d'un biais de genre, qui exige l'intervention de l'État. La modification majeure introduite par la loi dans le traitement juridico-institutionnel réside dans l'intégration d'une perspective préventive, et non simplement répressive, sur ce phénomène. Entre autres innovations de la loi, nous soulignons: (a) une définition étendue de la violence, qui inclut les violences psychologique, économique et morale, en plus des violences physique et sexuelle; (b) la création de services publics pluridisciplinaires d'accueil, y compris d'assistance sociale et de santé, tels que foyers et abris, services de santé spécialisés et centres spécialisés de la femme; (c) la détermination de mesures d'urgence pour la prévention des délits les plus graves et la protection des victimes; (d) l'augmentation du coût de la sanction pour l'agresseur ; (e) la création d'un tribunal unique compétent au civil et au pénal, plus adapté au caractère multidisciplinaire de la situation de violence ; (f) l'assistance juridique gratuite pour la femme victime, et pas uniquement pour l'agresseur ; $(\mathrm{g})$ le renforcement de l'autonomisation et des conditions de sécurité permettant à la victime de dénoncer les violences dont elle a fait l'objet, grâce à l'intégration des interventions de la police, du Parquet, des Défenseurs publics et des Tribunaux spéciaux en matière de répression et de prévention. ${ }^{15}$

11 La LMP a modifié de façon significative les termes du débat autour de la violence domestique dans le pays, en la faisant sortir des cercles d'experts pour en faire un important thème de discussion dans la sphère publique. Une de ses grandes réussites est, selon moi, d'une part, d'avoir largement fait passer le message selon lequel la violence contre les femmes n'est pas un crime mineur mais une grave violation des droits de l'Homme, et d'autre part, d'avoir favorisé l'autonomisation des femmes en leur donnant des moyens de réagir et de dénoncer la violence subie. Si la sousnotification des cas de violence reste une réalité difficile à changer, on enregistre, après l'entrée en vigueur de la loi, une augmentation des plaintes qui a permis de mieux dimensionner l'ampleur du problème au niveau national. Pour aider à prendre la mesure des avancées que l'on vient de décrire, on mentionnera le dynamisme des mouvements sociaux à partir de ce qu'on a appelé le printemps féministe de 2013, mais également, la contre-offensive à laquelle font face depuis peu les mouvements 
féministes, avec de violentes attaques contre ce qui est désigné péjorativement comme «l'idéologie de genre».

12 En effet, à partir de la LMP, nous avons pu commencer à discuter la violence contre les femmes et le sexisme en général à des niveaux autrement plus élevés qu'auparavant, permettant aussi l'émergence de critiques plus élaborées, comme celles proposées par le mouvement des femmes noires. Face à la vague conservatrice actuelle, la critique progressiste de la LMP doit être à la fois plus claire et plus affirmative, sans toutefois perdre de vue l'opportunisme des forces réactionnaires qui veulent revenir en arrière et annuler l'impact positif de la loi ${ }^{16}$. Heureusement, nous disposons aujourd'hui d'un corpus significatif d'évaluations de la mise en œuvre de la loi qui permet de définir des agendas progressistes pour ce combat.

13 En plus de la Mapa, l'Ipea (une importante structure de recherche en sciences sociales), a lui aussi publié une étude sur la LMP en 2015. L'Ipea a évalué l'effectivité de la loi à partir de l'évolution des chiffres d'homicides sur des femmes dans tout le pays. Si la loi ne traite pas directement du féminicide, il est permis de penser que lorsqu'il se propose d'intervenir sur le cycle de la violence intrafamiliale et domestique «là où il y a souvent exacerbation du degré d'agressivité, qui peut déboucher, et parfois débouche (souvent de façon inattendue) sur la mort de la conjointe », il « engendre aussi un effet de deuxième ordre qui réduit le nombre d'homicides pour motifs domestiques et de genre $\aleph^{17}$. Les résultats montrent que la LMP réduit de près de $10 \%$ le taux d'homicides à domicile contre les femmes par rapport au taux d'homicides contre des hommes dans les mêmes conditions ${ }^{18}$. En d'autres termes, la loi a ralenti l'augmentation du nombre d'homicides contre les femmes au Brésil. Les auteurs soulignent que la dynamique n'est pas uniforme sur l'ensemble du pays en raison des "différents niveaux d'institutionnalisation des services de protection des victimes de la violence domestique $»^{19}$. Là où les services spécialisés du Défenseur public, du Parquet et des commissariats de police, ainsi que les réseaux d'accueil, fonctionnent le mieux, ce taux a diminué dans des proportions plus significatives que là où ces services n'existaient pas ou ne fonctionnaient pas correctement.

14 Ces chiffres n'intègrent toutefois pas les critères raciaux et adoptent d'autres teintes quand on les compare à ceux de la Mapa, indiquant les écarts d'effectivité de la loi entre femmes blanches et femmes noires. L'absence de services satisfaisants, ayant un impact négatif sur les performances de la LMP, touche certaines populations avec plus d'intensité que d'autres. Les critères de race, de classe et de région (rurale ou urbaine, sud et sud-est ou nord et nord-est, etc.) sont déterminants dans cette analyse. La Mapa nous montre également que $62,7 \%$ des victimes de violence prises en charge dans les hôpitaux publics ont été agressées par des membres de leurs familles et leurs partenaires, ce qui est un indicateur de violence domestique, alors que les 37,3\% restants correspondent certainement à des femmes victimes de ce qu'on appelle la «violence courante ». Cette information n'est pas non plus disponible selon la race, ce qui nous prive de savoir dans quelles proportions ces situations se présentent de façon plus ou moins intense pour les blanches et pour les noires.

15 Pour ce qui est des services mis en place et bien qu'elle traite de l'intervention de différents organes de l'exécutif et de la justice, la loi est restée axée sur la réponse judiciaire apportée aux plaintes pour violence. Cela faisait sens dans un contexte où la judiciarisation des droits de l'Homme était un enjeu majeur au Brésil et dans le monde. L'expérience des dernières années ne permet plus, hélas, de porter une appréciation 
aussi optimiste sur l'arène judiciaire comme moteur de construction de la citoyenneté. Les divers diagnostics font le constat d'une application restrictive de la loi, bridant l'ambition de ses promesses ${ }^{20}$. En général, nous observons une prévalence de l'application des dispositifs répressifs et une démobilisation des dispositifs de nature préventive $^{21}$. Parmi les innombrables modalités de mesures de protection d'urgence prévues dans les différents articles de la loi, seules celles de nature pénale sont régulièrement mises en place. Bien que la liste des mesures prévues ne soit qu'indicative, permettant aux juges d'en employer d'autres non énoncées, nous observons qu'en réalité, les choses fonctionnent différemment. Seuls quelques états fédérés ont institué des tribunaux ayant compétence au civil et au pénal. Le réseau de protection des femmes - services de santé, foyers et formations - n'a jamais été correctement mis en place et il est aujourd'hui démantelé à grands pas ${ }^{22}$. Enfin, la transformation du délit de lésion corporelle légère en action pénale publique sans conditions, qui ne dépend pas du consentement de la victime et interdit l'application de la suspension conditionnelle du procès, continue de susciter la polémique au sein des groupes autant conservateurs que progressistes ${ }^{23}$. Le résultat est que la possibilité d'emprisonnement de l'agresseur est devenue la principale réponse de l'État à la violence domestique, et cela reste symboliquement très important, même si la LMP n'a pas fait augmenter la population carcérale brésilienne de façon significative ${ }^{24}$.

16 Ces critiques font planer le doute sur l'efficacité de la loi à l'égard du combat contre la violence de genre contre les femmes non-blanches et des milieux défavorisés. En effet, en dépit des insuffisances rappelées plus haut, la loi a permis de diminuer les cas de violence contre les femmes blanches, mais n'a pas eu le même effet pour la protection des femmes noires. Dans quelle mesure des lois comme la LMP, qui font appel à des catégories universelles comme "la femme», dans leur formulation et leur mise en œuvre, font-elles écho à la production d'études racialisées sur les privilèges des blancs et l'oppression sur les non-blancs? Eu égard à la répartition inégale des privilèges et des handicaps dans une société raciste, dans quelle mesure la femme noire victime de violence domestique a-t-elle été utile au féminisme hégémonique? Il s'agit donc maintenant de voir comment la racialisation des catégories de "genre" et de « violence domestique » permet de discerner plus clairement le contexte de la violence domestique contre les femmes noires.

\section{Racialisation du genre de la violence}

Le racisme et le sexisme, tout comme le classisme, sont des dimensions structurelles de la vie sociale et, comme telles, forgent des subjectivités et situent les individus socialement. En ce sens, noirs et blancs, hommes et femmes, sont constitués dans les rapports sociaux à partir de dispositifs de pouvoir qui, comme nous l'a enseigné Michel Foucault, échappent au contrôle individuel, tels que la discipline et la sexualité25. Ce processus de subjectivation ne se déroule toutefois pas de manière symétrique et les corps sont hiérarchisés à partir de la race et du genre, mais aussi d'autres marqueurs facilitant ou interdisant l'accès aux ressources matérielles et symboliques. Si nous ne prenons pas en compte cette inégalité quant à l'accès aux ressources permettant de vivre, à chaque fois que nous utilisons des stratégies « inclusives » et universalisantes, nous contribuons à des processus de subalternisation. 
18 C'est un phénomène du même ordre qui se produit dans le débat sur la violence domestique. Le féminisme international et brésilien s'est battu durant des décennies pour que les droits des femmes soient envisagés comme des droits de l'Homme et, dans cette lutte, la discussion autour de la violence domestique a gagné une importance majeure et a progressivement fait consensus parmi les divers mouvements féministes. La question de la violence domestique s'est donc universalisée en tant que problème de femmes. Cela témoigne de sa gravité, dans la mesure où la violence domestique constitue une des principales causes d'assassinat et de maladies incapacitantes des femmes. Mais dans ce combat, le féminisme "hégémonique » commence tout juste à reconnaître la composante raciale du problème qui détermine l'intensité et les spécificités avec lesquelles le phénomène affecte les corps.

19 Une des principales revendications de la pensée « décoloniale » est la décolonisation de la connaissance: la pensée hégémonique a imposé, avec violence, au monde périphérique et subalternisé, des catégories d'appréhension du monde qui sont venues désorganiser d'autres façons d'être au monde et ont mis en place des hiérarchies sociales rigides. Ces "violences épistémiques » ont engendré des classes de savoir et ont anéanti, totalement ou en partie, des cosmovisions originales et des classes de sujets, imposant aux plus subalternes le handicap d'avoir à « réapprendre » le monde à partir de la grammaire du colonisateur ${ }^{26}$. En ce sens, les violences épistémiques ont des conséquences profondes, autant en termes de rapports de pouvoir hiérarchiques, qu'en termes d'estime de soi des sujets subalternisés qui trouvent dans la «race» leurs limites et leurs mesures. En d'autres termes, une des tâches auto-imposées de la pensée " décoloniale » est la remise en cause des catégories épistémologiques édifiées à partir de la race.

20 Maria Lugones, dans son célèbre article « Vers un féminisme décolonial », affirme que «l'imposition coloniale du genre touche aussi les domaines de l'écologie, de l'économie, de gouvernement, et se lie au monde spirituel et à la connaissance ${ }^{27}$. Selon elle, l'entreprise coloniale elle-même a été menée à bien au moyen des catégories sexe et genre et on peut en voir la traduction dans le projet "civilisateur » européen de transformer les «bêtes", racialement marquées, que l'on trouvait encore ici-même dans les Amériques, en non-hommes et non-femmes. En effet pour Lugones, la dichotomie centrale de la modernité est celle qui distingue entre l'humain et le nonhumain et se réalise à partir de l'attribution de genre aux humains. Seuls les colonisateurs avaient un genre et étaient hommes et femmes, avec toutes les hiérarchies connues dans cette dichotomie. Aux colonisés-civilisés le genre était nié et on attribuait le sexe mâle ou femelle, dans une opération épistémique qui a permis ce qu'on pourrait appeler une "féminisation du sujet colonisé ", en d'autres termes, « l'accès brutal aux corps des personnes au moyen d'une exploitation inimaginable, du viol, du contrôle de la reproduction et de la terreur systématique $»^{28}$.

21 L'imposition du système sexe-genre a constitué l'un des outils de la destruction de cosmovisions qui ne s'organisaient pas nécessairement autour du sexe et du genre. L'imposition de ces catégories comme critère politiquement majeur de l'organisation sociale est ainsi une forme de violence épistémique dont les graves conséquences politiques et sociales demeurent d'actualité. L'analyse de Lugones montre à quel point l'élément de la race est fondamental pour comprendre jusqu'où un sujet peut avoir ou non un genre, dans quelle mesure certaines femelles racialement marquées sont des «femmes.» Plusieurs théoriciennes du féminisme noir dénoncent depuis longtemps 
l'impossibilité pour les hommes et les femmes noirs de s'inscrire dans les modèles de masculinité et de féminité créés à partir de l'expérience blanche européenne, et leur cantonnement corrélatif dans les catégories de «non-hommes » et de «non-femmes».

En effet, les stéréotypes de genre classiquement identifiés par le féminisme hégémonique pour illustrer les formes de naturalisation de l'oppression de genre ne s'appliquent pas de la même façon sur les corps des «non-femmes». Le mythe de la fragilité féminine, l'exigence de chasteté de la femme et la division sexuée du travail qui la confinait à la sphère privée, par exemple, n'opèrent pas également sur des corps blancs et sur des corps noirs. Lélia Gonzalez rappelait déjà que les femmes noires avaient toujours travaillé "aux champs ou dans les rues, comme vendeuses ou prostituées » et que leurs corps, marqués à la fois par le sexisme et par le racisme, mais aussi par le classisme, acquièrent une intelligibilité sociale spécifique, qui, dans le Brésil contemporain, se matérialise notamment dans les figures de la mulâtresse et de l'employée de maison ${ }^{29}$. De la même manière, le débat autour du contrat sexuel du travail, qui fait de l'homme un pourvoyeur de ressources et cantonnent la femme au foyer, ainsi que la lutte pour l'accès des femmes au marché du travail, prennent des contours très différents dès lors qu'il s'agit de femmes noires. Comme le dit Sueli Carneiro :

«... nous [femmes noires] faisons partie d'un contingent de femmes, probablement majoritaire, qui (...) n'ont rien compris quand les féministes se sont mises à dire que les femmes devaient sortir dans les rues et travailler $! »^{30}$.

Quant au débat sur la violence domestique, Tania Mara Campos Almeida et Bruna Cristina Jaquetto Pereira expliquent la façon dont les représentations spécifiques du corps de la femme noire fonctionnent pour autoriser les agressions dans le cadre du foyer :

"Ce sont des représentations qui guident des comportements et des pratiques violentes contre les femmes noires et mulâtresses de la part de leurs compagnons et qui englobent : un contrôle permanent sur leur sexualité, dans la mesure où elles sont considérées hyper-sexualisées; la négation de leur sexualité, car leurs attributs esthétiques s'éloignent de ceux des femmes blanches, considérées canon de beauté; la violence sexuelle, comme forme d'humiliation et/ou la déconsidération de leur humanité; les humiliations dégradantes, avec ou sans insulte raciale, ancrées dans la perception de leur statut socialement subalterne; l'exploitation économique des ressources obtenues par leur travail rémunéré, fondée sur l'imaginaire qui fait d'elles des travailleuses infatigables et que le simple fait d'avoir des rapports avec elles constitue en soi une faveur qui doit être rétribuée ; l'exploitation de leur travail dans le cadre domestique, qui s'appuie sur l'imaginaire qui fait d'elles des personnes naturellement dédiées au foyer; l'agression physique brutale, qui part du postulat de leur grande force physique $»^{31}$.

L'étude de Bruna Cristina Jaquetto Pereira relève des aspects de la catégorie "genre " qui ne se révèlent que si l'on adopte une perspective épistémologique et politique racialisée. D'un côté, «il reste » beaucoup de vie au-delà du genre : l'expérience de la violence brutale sur les corps de ces femmes ne s'explique pas seulement par le fait qu'elles sont du sexe féminin. Mais nous soulignons ici la façon dont Pereira met à nu la race de la catégorie genre elle-même :

«Il ne s'agit pas de penser de manière générique pour ensuite prendre en compte, en sus, de possibles offenses raciales envers des femmes noires. Si on a pu identifier l'inscription de la race/couleur dans la violence domestique contre les femmes, l'étape suivante doit être de chercher à comprendre la nature même de cette mobilisation, plutôt que de supposer que de possibles offenses raciales constituent 
des offenses ou des violences venant s'ajouter à celles rencontrées à l'égard des

femmes blanches ou des femmes en général $»^{32}$.

À partir des entretiens de 14 femmes qui se déclarent elles-mêmes noires et mulâtresses, ayant subi des violences exercées par leurs (anciens) maris / (anciens) compagnons à Brasilia, Pereira s'est penchée sur la façon dont le racisme donne forme à l'expérience de ces femmes dans trois directions: (a) les expériences antérieures de violence, dans le cercle familial proche, qui structurent l'univers interprétatif du vécu de la violence domestique actuelle dénoncé par les enquêtées; (b) les contextes de violence psychologique/morale qu'elles relatent; et (c) le type de réaction aux agressions subies ${ }^{33}$. Pereira en conclut qu'il existe des formes de manifestation explicite $\mathrm{du}$ racisme dans les rapports de certaines de ces femmes à leurs agresseurs, sans que cela ne soit perçu comme de la violence domestique. Elle identifie également des dynamiques entre victime et agresseur qui ne peuvent être correctement comprises qu'en mobilisant la catégorie analytique de la race pour penser les rapports de genre. D'après Pereira, il y a toute une série d'attentes vis-à-vis du comportement de ces femmes racialement marquées, mais «si l'on ne prend en compte que la condition de genre, il devient difficile de comprendre les origines et les significations de ces attentes, qui semblent s'éloigner des représentations considérées traditionnelles. $»^{34}$

Nous discuterons maintenant les risques encourus par les luttes en faveur de politiques publiques coordonnées, menées à partir de catégories universelles, ainsi que les brèches par lesquels le racisme parvient à s'infiltrer. Nous mobiliserons ici les théories de l'intersectionnalité qui, dans le combat des femmes noires et d'autres femmes non blanches pour révéler les formes de domination au sein du féminisme, se sont révélées constituer une importante clé d'analyse et de dénonciation de l'invisibilisation des corps et des inégalités dans la distribution de la précarité.

\section{La production de l'invisibilité de la femme noire dans la lutte contre la violence domestique}

Dans la lignée de la critique poststructuraliste des identités essentialisées et homogènes, les théories de l'intersectionnalité annoncent de nombreuses manières $d^{\prime}$ ' être femme» et $d^{\prime}$ ' être une personne noire $»^{35}$. Les femmes noires vivent le sexisme et le racisme d'une manière singulière, qui n'est pas immédiatement accessible à des femmes blanches. Ainsi, quand il parle au nom de la femme, en tant que catégorie universelle et uniforme, le féminisme court le risque de permettre des formes de domination intragroupe, où les femmes des races et des classes dominantes reproduisent à l'encontre des femmes subalternisées des formes de domination semblables à celles qu'elles dénoncent dans le patriarcat. Pour Kimberle Crenshaw, de tels processus adviennent dans le cadre d'opérations de sur-inclusion ou de sousinclusion. Les premières se produisent lorsqu'un problème qui touche exclusivement ou de façon disproportionnée un sous-groupe de femmes est « absorbé par la structure de genre, sans aucune tentative de reconnaissance du rôle que le racisme ou toute autre forme de discrimination peut avoir joué dans de telles circonstances $»^{36}$. En conséquence, les particularités du problème ne sont plus prises en compte et les politiques publiques le concernant perdent de leur efficacité. Les opérations de sousinclusion, quant à elles, sont des situations où un problème touche un sous-groupe de 
femmes, mais « n'est pas perçu comme un problème de genre, parce que les femmes des groupes dominants n'en ont pas l'expérience $»^{37}$.

Nous entendons que dans la lutte contre la violence de genre au Brésil, on a vu à l'œuvre deux formes d'invisibilisation des femmes noires, mais aussi d'autres femmes non-blanches. Il y a eu sur-inclusion, dans la mesure où les spécificités du problème de la violence domestique contre les femmes noires n'ont pas été correctement prises en compte dans les choix qui ont orienté la loi. Nous songeons ici en particulier à la survalorisation des actions de la justice lors de l'élaboration de la $\operatorname{loi}^{38}$ et du positionnement des militantes qui, tout en affirmant l'importance de la prévention, ont investi la défense de l'emprisonnement comme symbole contre la banalisation du délit de violence domestique ${ }^{39}$. En revanche, il y a eu sous-inclusion des femmes noires dans la lutte contre la violence sur le corps féminin, dans la mesure où, si le but du féminisme est d'émanciper toutes les femmes, nous aurions dû intégrer à l'agenda féministe lié à la violence de genre, les situations de violence non domestique qui touchent des milliers de femmes noires. Dans ces deux modes d'invisibilisation, la femme noire a été instrumentalisée : elle apparaît (quand elle apparaît) comme le symbole de la victime de violence domestique, mais ensuite elle n'est plus entendue en tant qu'actrice de son propre combat émancipateur. On approfondira ce point plus bas.

Pour en revenir à la sur-inclusion, dans l'un de ses articles fondateurs, Crenshaw démontre, données et situations empiriques à l'appui, les aspects intersectionnels structurels qui caractérisent la violence domestique subie par les femmes noires à Los Angeles. L'auteure soutient que les efforts des féministes pour construire la violence domestique comme un problème de toutes les femmes, de toutes les races et couches sociales, ont abouti à une certaine sur-inclusion, allant jusqu'à réaffecter des crédits à des politiques publiques, comme l'assistance juridique, qui ne répondaient pas vraiment aux besoins les plus pressants des femmes noires. La reconnaissance du fait que la violence domestique n'est pas un problème exclusif de communautés racialement et ethniquement marquées, dit-elle, est devenue affirmation de ce qu'elle touche toutes les femmes de façon égale, ce qui ne correspond pas à la réalité ${ }^{40}$. Certaines femmes font face à des obstacles disproportionnés. Crenshaw explique, par exemple, que la plupart des femmes qui cherchent un accueil dans un foyer sont au chômage ou sousemployées, et la question financière est la première barrière à lever pour briser le cycle de la violence. D'autant que le réseau privé de soutien de ces femmes tend à être plus précaire : comme le chômage est plus important dans la population noire, les femmes noires ont encore moins de chances que les femmes blanches d'obtenir un soutien financier de la part d'amis ou de parents. Pour échapper à la violence, elles ont besoin de politiques publiques de redistribution, comme des foyers dignes, des programmes de formation et de placement professionnel et de crèches pour leurs enfants ${ }^{41}$.

Nous pouvons formuler, grosso modo, les mêmes réserves à l'égard de la LMP. Tant au niveau de la prévention que de la répression, le premier rôle est dévolu à la justice au détriment des autres acteurs. Comme nous l'avons vu à la partie 1 , seuls deux articles de la loi traitent du réseau d'accueil de ces femmes, qui requiert la participation des administrations municipales, des Etats fédérés et de l'Etat fédéral. En outre, les services mentionnés par ces articles n'ont jamais été suffisamment mis en place et sont aujourd'hui en cours de démantèlement accéléré. Il en va de même d'ailleurs pour les services de la justice: on ne trouve pas dans tous les Etats un bureau du Défenseur 
public correctement préparé pour accueillir les victimes, ce qui affaiblit leurs possibilités d'intervention active dans un procès.

31 Toujours sur le choix de la voie judiciaire, rappelons que les dispositifs qui étendaient la garantie de protection des femmes contre des formes de violence autres que physique et sexuelle ont été peu mobilisés, une fois de plus au détriment prépondérant des femmes noires. Les aspects économiques de la violence domestique sont peu reconnus et rares sont les procès liés à la violence dite patrimoniale. La lecture, dans une perspective intersectionnelle, des récits inclus aux 187 procès concernant des femmes noires et défavorisées nous a permis d'identifier cinq contextes récurrents (la liste n'est pas exhaustive) qui déclenchent la violence contre les femmes noires de milieux défavorisés :

«(i) appropriation des biens de ces femmes par leurs agresseurs, (ii) menaces et agressions sur le lieu de travail des femmes, (iii) conflits liés à la garde des enfants, (iv) conflits autour de la propriété des lieux de résidence des femmes, et (v) conflits liés à la dépendance chimique des agresseurs $»^{42}$.

Dans toutes ces affaires, l'État a ignoré le fait évident que les conditions matérielles et économiques de ces femmes ont été déterminantes dans l'intensité de l'agression qu'elles ont subie. Or la nomenclature juridique employée par la police et par la justice laisse de côté des aspects essentiels de la situation de violence à laquelle elles sont soumises. Par exemple, Nadir (nom fictif), après plusieurs années de violence domestique, a quitté son mari qui, depuis, ne cesse d'entrer chez elle et d'y dérober ses biens, comme le réfrigérateur et la cuisinière, pour les revendre. La façon dont l'État a transformé la situation dans le discours judiciaire est surprenante :

«... la nomenclature choisie est celle d'exercice arbitraire de raisons personnelles (art. $354 \mathrm{CP}$ ), type pénal qui requiert une prétention légitime à un recouvrement, dans le cas présent, une prétention à caractère financier. Aucun procès-verbal ne mentionne toutefois quelque dette que ce soit de la victime envers l'agresseur $»^{43}$.

33 Au-delà de la question de la qualification du délit, illustrée ci-dessus, se pose également celle des types de mesures de protection sollicitées (par l'autorité policière, au nom de la victime) et accordées par le tribunal. Nous nous rendons compte ici que la capture du mécanisme des MPU par la logique pénale a des conséquences encore plus graves, pouvant aller jusqu'à l'inefficacité totale de la protection. Pour illustrer cette double forme d'invisibilisation de la précarité accrue à laquelle sont exposées ces femmes (par la qualification du délit et la concession des MPU), nous prendrons le cas de Claudia (nom fictif) qui, après avoir demandé de l'argent à son compagnon et père de leurs filles, est agressée dans la rue :

«Bien que le déclencheur de l'agression soit lié à la subsistance familiale, n’a pas été prononcée (ni même demandée d'ailleurs) la mesure prévue à l'alinéa $\mathrm{V}$ dudit dispositif légal, des aliments. Bien que l'intégrité physique de Claudia soit formellement protégée [par la MPU d'éloignement], son exposition à la violence, en conséquence de nécessité de contact pour son alimentation et celle de sa fille, reste identique. La qualification des faits sur la base de la lésion corporelle (art.129 CP) laisse dans l'ombre l'aspect patrimonial de la violence, de sorte que la réponse juridique (éloignement de l'agresseur) semble, dans la meilleure des hypothèses, impuissante à faire cesser la violence $»^{44}$.

34 Un schéma récurrent a attiré notre attention dans l'étude élargie, après l'analyse des 355 procédures. Le plus grand obstacle à la mise en œuvre des MPU est la remise des assignations de justice. De façon très cohérente, les trois tribunaux étudiés respectaient le délai de $48 \mathrm{~h}$ pour recevoir (ou rejeter) les mesures puis expédier les mandats, prévu à 
l'art. 18 de la loi, mais de nombreuses assignations n'étaient pas remises et, la plupart de celles qui l'étaient effectivement, l'étaient dans un laps de temps considérable entre l'expédition et la remise ${ }^{45}$. En général, la justification, toujours brève, de ce délai ou de la non-réalisation était que le prévenu ou son adresse n'avait pu être localisé. Il suffit de croiser les données des assignations à l'information sur le profil des personnes généralement impliquées dans les affaires dont ces tribunaux ont à connaître pour avoir une première démonstration concrète de l'injuste répartition de la précarité et de l'exposition à la violence, liée à la question du logement et de la mobilité urbaine. Nous savons pertinemment que les représentants de l'État n'entrent pas dans les quartiers défavorisés, si ce n'est dans le cadre d'opérations de police, et la difficulté à remettre les assignations peut être vue comme une expression supplémentaire de cette réalité.

L'enquête de Marilia Montenegro identifie également certains aspects spécifiques de la violence domestique contre les femmes de milieux défavorisés. À partir d'une étude empirique dans la ville de Recife, initialement dans le cadre d'un tribunal spécial pénal et, par la suite (suite à l'entrée en vigueur de la LMP) dans celui d'un tribunal exclusivement compétent en matière de violence domestique, elle souligne l'importance du critère de classe dans le recours aux instances judiciaires. Elle signale que de nombreuses affaires civiles n'arrivaient pas à ces tribunaux, dans la mesure où l'accès à la justice de nature civile et, en particulier, familiale, est plus difficile pour les plus pauvres. Les coûts élevés, que nécessite un avocat, l'insuffisance ou l'inexistence de défenseurs publics, des rites formels et lents, sont des obstacles plus important devant les tribunaux spécialisés en droit de la famille que devant les tribunaux spécialisés contre la violence domestique. Ainsi les problèmes liés à des séparations, comme le partage des biens, la fixation des charges destinées à couvrir les besoins des enfants, etc., arrivent en justice par la voie des commissariats de police, pour les ménages pauvres, et non par celle des tribunaux de la famille. L'informalité de la vie dans les banlieues place ainsi leurs habitants hors de portée du droit de la famille : les logements et terrains sans titre de propriété, les emplois non déclarés, par exemple, sont des entraves au partage des biens et à la fixation des pensions alimentaires ${ }^{46}$.

Mais les femmes noires ont été sous-incluses de bien d'autres façons encore. L'une d'elles, analysée à la partie précédente, est la non-identification des offenses racistes en tant que forme de violence psychologique dans le cadre de la LMP, tant pour ce qui est de la qualification du délit que pour les services proposés par les tribunaux, comme les groupes de réflexion des agresseurs ${ }^{47}$. Dans notre étude élargie, nous n'avons trouvé dans aucun des dossiers analysés de récits d'insultes et/ou d'autres formes de violence fondées sur la race. Il est permis de penser que cette absence est due autant à la difficulté des victimes à qualifier en ces termes la violence subie, qu'à la façon dont elles sont accueillies par les agents de police qui orientent les dépositions à partir de ce qu'ils jugent le plus pertinent dans le cadre de la violence domestique. Les deux situations sont des manifestations d'un racisme structurel profond.

Ana Flauzina analyse les insuffisances de la LMP à partir des dynamiques entre " résistance » et " militantisme ${ }^{48}$. Elle affirme que, dans les moments importants, des choix militants ont abouti à la mise sous silence de la résistance, essentiellement, des femmes noires et pauvres. Il y a, d'après elle, une superposition de silences, une pratique que la loi Maria da Penha n'a pas été capable de régler. Pour Flauzina, la dénaturalisation des crimes de violence domestique et la soumission des agresseurs à une procédure de responsabilité juridique ${ }^{49}$ obtenues avec la promulgation de la LMP, 
sont des victoires importantes des militantes féministes, face à un discours toujours puissant de banalisation de la violence contre les femmes. Mais d'après elle, les militantes concentrent leurs efforts sur l'objectif de faire tomber les obstacles aux poursuites contre les agresseurs, plutôt que sur la valorisation des souhaits des victimes. Son grand exemple est celui des restrictions à la possibilité de rétractation des victimes, possibilité qui empêcherait que le procès pénal aille de l'avant. La justification du féminisme hégémonique à une telle restriction est la difficulté à parler d'autonomie de la victime dans des cadres d'oppression systématique; l'idée est donc de mettre la victime à l'abri des pressions destinées à la faire renoncer au procès qu'elle intente. Flauzina souligne que les militantes n'ont pas choisi de se battre pour de meilleures conditions pour la manifestation de la rétractation (avec par exemple une assistance juridique et émotionnelle adéquate) ou pour des conditions matérielles pour exercer ce choix de manière autonome. En ce sens, selon Flauzina, la victime a été instrumentalisée : son cri de dénonciation est utile pour déclencher le procès, mais après cela, sa voix n'est plus considérée comme autonome ${ }^{50}$. Même s'il importe évidemment de tenir compte de toute la complexité qu'emporte le complexe contexte de fragilité des femmes en situation de violence, l'objection de Flauzina soulève d'importantes questions.

Enfin, il est une autre forme de sous-inclusion résultant de la manière dont le féminisme hégémonique prend en compte la lutte contre la violence contre les femmes: c'est la mesure dans laquelle les environnements spécifiques de violence auxquels les femmes noires sont exposées sont négligés. L'agenda de la lutte contre la violence de genre s'est concentré sur les aspects liés à la violence domestique et a systématiquement exclu les questions liées à ce qu'on appelle la "violence courante». Toutefois, un nombre significatif de femmes assassinées et agressées l'ont été dans un cadre autre que domestique. Or nous savons que les femmes noires sont structurellement plus exposées que les autres à des conditions de précarité aigüe, induites politiquement, et qu'elles sont à ce titre surexposées à la violence induite par le trafic de drogues et la répression policière. Elles prennent aussi plus de risques que les blanches au moment de tirer au clair les circonstances de l'exécution sommaire de leurs partenaires et de leurs enfants. Ces contextes marquent violemment leurs corps et leurs vies. Le caractère racial de la violence d'Etat est bien connu ; mais sa dimension de genre est moins discutée, sans doute parce qu'il concerne avant tout les femmes noires. Les mêmes codes de genre liés à la masculinité et qui structurent le sexisme, alliés aux codes de la blanchitude, engendrent la situation actuelle de violence d'Etat contre les corps noirs. Mais la question apparaît rarement à l'ordre du jour du féminisme hégémonique parce qu'elle est perçue comme un problème qui concerne les hommes (pauvres et noirs). Depuis des décennies, les féminismes noirs attirent pourtant l'attention sur cette mise sous silence et cette invisibilisation, et exigent des féministes qu'elles s'investissent sur cette cause :

«Le racisme ne pose pas problème simplement parce que les militantes féministes blanches seraient en fait racistes, individuellement. Elles représentent un faible pourcentage des femmes dans cette société. Quand bien même elles seraient toutes antiracistes depuis le début il faudrait encore faire de l'élimination du racisme une question féministe centrale $»^{51}$.

La blanchitude a donc participé à l'établissement de critères servant à déterminer quelles sont les vies vivables. Pourtant, comme condition structurante de l'expérience de l'oppression de la majorité des femmes sur la planète, la lutte contre le racisme 
devrait être un des axes du féminisme. Ici apparaît un enjeu supplémentaire : comment parvenir à un tel résultat sans usurper le discours des femmes victimes des processus de subalternisation aigüe?

\section{Conclusion. Sur les discours et l'écoute : des pistes pour la lutte contre la violence de genre}

40 L'avertissement adressé par Spivak aux intellectuels qui prétendent parler pour les autres opprimés nous aide à comprendre aussi les dilemmes qui structurent le militantisme et la résistance subalterne. Dans un monde marqué par les opérations de violence épistémique, aucun discours de résistance ne peut être prononcé hors du discours hégémonique. Si l'on ne parvient pas à modifier en profondeur cet ordre des choses, l'intellectuelle et la militante resteront vouées à constituer perpétuellement la victime de violence domestique comme un objet de connaissance et non comme un acteur de sa propre émancipation, et à reproduire les structures de pouvoir et d'oppression contre lesquels elles s'insurgent en théorie. La tâche qui s'impose est donc de s'atteler, à un niveau épistémique, à changer les conditions de production de la subalternité et, à un niveau politique, à modifier l'infrastructure injuste et inductrice comme distributrice de la précarité des corps. Cela suppose, en amont, la construction de ce que Spivak appelle une "infrastructure d'écoute", sans laquelle la voix de la victime de violence est passée sous silence face au mégaphone des puissants. Lutter contre les structures productrices de la subalternité semble surtout vouloir dire construire une « infrastructure d'écoute » capable d'aller jusqu'au subalterne.

41 Ce que cela signifie pour la lutte contre la violence de genre contre les femmes n'est pas définissable péremptoirement. Les stratégies et les alliances de pouvoir sont changeantes et les stratégies anti-hégémoniques doivent l'être elles aussi. J'admets que la LMP peut être un cadre normatif sur lequel s'appuient des infrastructures d'écoute et d'insubordination, à condition que l'académie et les militants se prêtent à une reconstruction profonde et ouverte à l'innovation. Sans prétendre régler le problème, je crois que l'objectif majeur doit être d'aller vers les réformes nécessaires pour que les victimes de violence soient valorisées en tant qu'actrices de leurs propres démarches pour surmonter la violence, avec tout l'appui nécessaire pour ne pas être laissées dans la précarité. En effet nous devons, théoriciennes et militantes, investir dans un réseau d'accueil sans doute plus que dans un rôle de surveillance de la justice, ainsi que dans une assistance juridique compétente et gratuite quand la voie judiciaire est pertinente.

Rien de cela ne se fera sans la présence des sujets mêmes dont les corps sont systématiquement brutalisés. La représentation de ces sujets dans les espaces de pouvoir, que ce soit au sein de l'État, à l'université ou dans la société civile, n'est pas négociable si nous voulons vraiment ébranler un tant soit peu les conditions de production de la subalternité. Vis-à-vis des femmes noires et autres non-blanches, particulièrement subalternisées dans des sociétés sexistes et racistes comme les nôtres, les revendications féministes ne peuvent négliger le racisme en tant que catégorie de compréhension de l'oppression et des marques de violence sur les corps des femmes. Cela signifie, en premier lieu, un auto-examen critique permanent des privilèges de la blanchitude dont les intellectuelles et les militantes ne sont pas à l'abri. 


\section{BIBLIOGRAPHIE}

ALMEIDA, Tania Mara Campos, PEREIRA, Bruna Cristina Jaquetto, "Violência Doméstica e Familiar contra mulheres pretas e pardas no Brasil : reflexão pela ótica dos estudos feministas latinoamericanos", In Critica e Sociedade : Revista de cultura política, V.2, n.2, Dossiê : Cultura e Política, dez 2012, pp. 58-59.

BARSTED, Leila Linhares, "Lei Maria da Penha : uma experiência bem-sucedida de advocacy feminista”, In : CAMPOS, Carmen Hein (Org.), Lei Maria da Penha-Comentada em Uma Perspectiva Jurídico- Feminista, Rio de Janeiro : Lumen Juris, 2011, p. 13-42.

BEAUVOIR, Simone, O segundo sexo, São Paulo : Difusão Europeia do Livro. 1967.

BERNARDES, Márcia Nina ; COSTA, Rodrigo de Souza, "Os parâmetros Internacionais de Prevenção da Violência Doméstica contra as Mulheres : uma comparação com os instrumentos previstos na Lei Maria da Penha", In : Cecilia Caballero Lois, Daniela da Rocha Brandao, Samantha Ribeiro Meyer-pflug. (Org.). Direito Internacional dos Direitos Humanos 1. 1ed. Florianópolis : Conpedi 2015, 2015, v. 1, pp. 358-384.

BERNARDES, Márcia Nina, “Aspectos Transnacionais da Lei Maria da Penha”, Direito, Estado e Sociedade (Impresso), v. 45, p. 20-40, 2014.

BERNARDES, Márcia Nina, "Domestic Violence and Gender Oppression: an Analysis of Brazilian Law in Light of a Theory of Democratic Justice". In: Marek Zirk-Sadowski, Bartosz Wojciechowski, Karolina M. Cern. (Org.), Towards the Recognition of Minority Groups: Legal and Communication Strategies, 1ed. Londres: Ashgate Publishing Ltd, 2014, v. 1, p. 1.

BERNARDES, Márcia Nina, "Philosophical and Jurisprudential Issues on Domestic Violence and Gender Discrimination”, In: Marcelo Galuppo; Monica Sette Lopes; Lucas Montijo; Karine Slagado; Thomas Bustamante. (Org.), Human Rights, Rule of Law and the Contemporary Social Challenges in Complex Societies, Proceedings of the XXVI World Congress of Philosophy of Law and Social Philosophy. 1ed.Belo Horizonte : Initia Via, 2015, v. 1, p. 50.

BERNARDES, Márcia Nina ; ALBUQUERQUE, Mariana Imbelloni Braga. Violências Interseccionais silenciadas em Medidas Protetivas de Urgência / Intersectional Violence silenced in Judicial Proceedings. REVISTA DIREITO E PRÁXIS, v. 7, p. 01-26, 2016.

BERNARDES, Márcia Nina ; COSTA, Rodrigo de Souza, "Medidas Protetivas de Urgência e Violência Contra as Mulheres : análise da aplicação da aplicação da Lei Maria da Penha no Juizado de Violência Doméstica e Familiar contra a Mulher de Niterói”, In : Marcos Alves Da Silva ; Silvana Beline Tavares. (Org.). Gênero, sexualidades e direito I., 1ed. Florianópolis : Conpedi 2016, 2016, v. 01, pp. 64-84.

BERNARDES, Márcia Nina ; COSTA, Rodrigo de Souza. ; OLIVEIRA, Adriana Vidal de. Violência Doméstica, discriminação de gênero e medidas protetivas de urgência ; 1. ed. Curitiba : Juruá, 2016. v. 01. $138 \mathrm{p}$.

BERNARDES, Márcia Nina ; OLIVEIRA, Adriana Vidal de. "As medidas protetivas de urgência : perfil dos procedimentos em tramitação no juizado de violênciia doméstica e familiar da comarca de Duque de Caxias", In : Juvêncio Borges Silva ; Tereza Cristina Sorice Baracho Thibau ; Edinilson Donisete Machado. (Org.). Acesso à justiça I., 1ed.Florianópolis : Conpedi 2015, 2015, v. 1, pp. 434-452. 
BUTLER, Judith, Notes toward a Performative Theory of Assembly, Cambridge: Harvard University Press, 2015.

CAMPOS, Carmen Hein. Lei Maria da Penha, "a necessidade de um novo giro paradigmático", Revista Brasileira de Segurança Pública, São Paulo, v. 11, n. 1, 10-22, Jan-Fev. 2017.

CARNEIRO, Sueli, "Enegrecer o feminismo : a situação da mulher negra na América Latina a partir da perspectiva de gênero".

COMISSÃO PARLAMENTAR MISTA DE INQUÉRITO, Relatório Final. Brasília : 2013.

COSTA, Rodrigo de Souza ; OLIVEIRA, Adriana Vidal de., "LEI 11.340/06 E SISTEMA PENAL : O QUÃO PUNITIVOS SÃO OS JUIZADOS DE VIOLÊNCIA DOMÉSTICA E FAMILIAR CONTRA A MULHER", In : Juliana Teixeira Esteves ; José Luciano Albino Barbosa ; Pablo Ricardo de Lima Falcão. (Org.). Direitos, gênero e movimentos sociais II. 1ed.Florianópolis : CONPEDI, 2014, v. 1, pp. 267-282.

CRENSHAW, Kimberlé, "Documento para encontro de especialistas em aspectos da discriminação racial relativos ao gênero", Revista de Estudos Feministas, 10 (1). 2002.

CRENSHAW, Kimberlé, “Mapping the Margins: Intersectionality, Identity Politics, and Violence against Women of Color”, In: Stanford Law Review. vol. 43, n 6 (jul., 1991).

FLAUZINA, Ana Luiza Pinheiro. "Lei Maria da Penha : entre os anseios da resistência e as posturas da militância”, A. Flauzina, F. Freitas, H. Vieira, T. Pires (orgs), Discursos Negros : legislação penal, política criminal e racismo, Brasília, Brado Negro. 2015. p. 126.

FOUCAULT, Michel., História da Sexualidade - a vontade de saber, vol. 1.a ed. São Paulo, Graal, 2001.

FRIEDAN, Betty, The Feminine Mystique, New York : W.W. Norton \& Company, Inc. 1963.

GONZALEZ, Lélia, "Racismo e Sexismo na Cultura Brasileira”, In : Revista Ciências Sociais Hoje. ANPOCS, 1984.

HOOKS, Bel, Feminist Theory: from margins to center, South End Press. 1984.

IPEA, Avaliando a efetividade da Lei Maria da Penha, Brasília. 2015.

LUGONES, Maria, “Rumo a um feminismo descolonial” In : Estudos Feministas 22(3) : 935-952, setembro-dezembro/2014.

MEYERSFELD, Bonita, Domestic Violence and International Law, Oxford: Hart Publishing, 2010.

MONTENEGRO, Marilia, Lei Maria da Penha : uma análise criminológico-crítica, Rio de Janeiro : Revan, 2015.

PASINATO, Wânia, "10 anos de Lei Maria da Penha. O que queremos comemorar ?", Sur. Revista Internacional de Direitos Humanos (Impresso), v. 1, p. 100, 2017.

PEREIRA, Bruna Cristina Jaquetto, Tramas e dramas de gênero e de cor : a violência doméstica contra mulheres negras. Brasília : Brado Negro, 2016.

PIRES, Thula Rafaela de Oliveira, "Direitos humanos traduzidos em pretueguês". In :

Transformações, Conexões e Deslocamentos. Seminário Internacional Fazendo Gênero 11 \& 13th Women's Worlds Congress (Anais Eletrônicos), Florianópolis, 2017, ISSN 2179-510X.

PIRES, Thula, “O que significa renunciar a uma categoria ?”, In : Empório do Direito. 2017.

SCHUCMAN, Lia, "Entre o "encardido", o "branco" e o "branquíssimo" : Raça, hierarquia e poder na construção da branquitude paulistana.", Tese de Doutorado, Psicologia Social, Universidade de São Paulo, 2012. 
SPIVAK, Gayatri, Pode o Subalterno Falar ?, Belo Horizonte : Editora UFMG, 2010.

WEISELFISZ, Julio Jacob, Mapa da Violência : homicídio de mulheres no Brasil. FLACSO Brasil. 2015.

\section{NOTES}

1. Intervention de Jurema Werneck, fondatrice de l'ONG Criola et, depuis 2017, Directrice d'Amnesty International au Brésil, lors des 2èmes journées de dialogue national sur la violence domestique.

2. S. CARNEIRO, Dossiê Mulheres Negras e Violência Doméstica, São Paulo, Geledés, 2017, p. 33.

3. J. J. WEISELFISZ, Mapa da Violência : homicídio de mulheres no Brasil, FLACSO Brasil, 2015.

4. T. R. de O. PIRES, « O que significa renunciar a uma categoria ?», Empório do Direito, 2017.

5. S. BEAUVOIR, O segundo sexo. São Paulo, Difusão Europeia do Livro, 1967.

6. T. R. De O PIRES, « Direitos humanos traduzidos em pretueguês ", Transformações, Conexões e Deslocamentos. Seminário Internacional Fazendo Gênero 11 \& 13th Women's Worlds Congress (Anais Eletrônicos), Florianópolis, 2017.

7. Sur les conditions de la création de la subalternité et sur le rôle des intellectuels, voir G. SPIVAK, Pode o Subalterno Falar ?, Belo Horizonte, Editora UFMG, 2010.

8. L. SCHUCMAN, “Entre o 'encardido', o 'branco' e o 'branquíssimo' : Raça, hierarquia e poder na construção da branquitude paulistana", Thèse de Doctorat, Psychologie Sociale, Université de Sao Paulo, 2012 : 'It is important to underscore 'whiteness' as a relational category. The 'white' elite in latin-American countries may not be perceived as such in Europe and NorthAmerica. The privileges that come with the recognition as a white person may also shift in different settings where one is displaced from the position of a 'Universal subject' and is perceived as a latin American woman or man".

9. A. L. FLAUZINA, "Lei Maria da Penha: entre os anseios da resistência e as posturas da militância”, A. L. FLAUZINA, F. FREITAS, H. VIEIRA, T. PIRES (eds.), Discursos Negros : legislação penal, política criminal e racismo, Brasília, Brado Negro, 2015, p. 126.

10. Dans cette analyse, outre la Mapa, déjà mentionnée, j'utilise d'autres études récentes sur la LMP, menées par l'IPEA et par les ONGs Criola et Geledés, CERQUEIR Daniel, MATOS Mariana. Voir A. P. MARTINS, J. P. AVALIANDO, A efetividade da Lei Maria da Penha, Brasília, IPEA, 2015. J. WERNECK, DOSSIE GELEDES CRIOLA, A situação dos direitos humanos das mulheres negras no Brasil: violências e violações, Rio de Janeiro et São Paulo, 2016; S. CARNEIRO, Dossiê Mulheres Negras e Violência Doméstica, São Paulo, Geledés, 2017.

11. M. N. BERNARDES, R. S COSTA, A.V. OLIVEIRA, Violência Doméstica, discriminação de gênero e medidas protetivas de urgência. 01. ed. Curitiba : Juruá, 2016. Nous voulions savoir, entre autres, quelle réponse apportait l'État à la première approche de la justice par une victime de violence domestique, comment était enregistré le type de violation qu'elle avait subie, quelles mesures demandait la victime et lesquelles étaient effectivement prises en compte, quel type de preuve figuraient au dossier et, enfin, quel type de preuve était considéré comme suffisant, au moins pour une protection d'urgence. Nous voulions discuter certaines questions épistémologiques et politiques liées à la construction discursive du profil des victimes de violence domestique, la mesure prioritaire que l'État accorde à la protection de cette victime par rapport à d'autres biens juridiques (comme la propriété) et la crédibilité du récit de la victime comme pièce à conviction justifiant la concession de cette mesure.

12. M. N BERNARDES, M. I. ALBUQUERQUE, "Violências interseccionais silenciadas em medidas protetivas de urgência”, Revue Direito e Práxis, vol. 7, 2016. 
13. Voir L. L. BARSTED, "Lei Maria da Penha: uma experiência bem-sucedida de advocacy feminista", in C. H. CAMPOS (Eds.), Lei Maria da Penha - Commentée dans Uma Perspectiva JurídicoFeminista, Rio de Janeiro, Lumen Juris, 2011, pp.13-42. Sur les actions au niveau national et international autour de l'approbation de la loi Maria da Penha, avec l'historique de cette question dans l'agenda du féminisme brésilien et international, voir M. N. BERNARDES, "Aspectos Transnacionais da Lei Maria da Penha", Revista Direito, Estado e Sociedade, vol. 45, 2014, pp. 20-40.

14. Dans un cadre général, comme problème touchant toutes les femmes (mais pas de la même manière), je soulignerais le caractère éminemment domestique de cette forme de violence : victime et agresseur se connaissent, sont dans une relation qui donne lieu à des sentiments divers et contradictoires, vivent dans les mêmes espaces et ont des parents communs. La violence domestique ne se manifeste pas lors d'un seul épisode, mais dans une situation continue qui peut se prolonger et connaître des intensités et des contours différents dans le temps. Les stéréotypes de genre qui, d'un côté, cantonnent les femmes dans les tâches de garde des enfants et des personnes âgées chargée de préserver l'harmonie du foyer, et de l'autre, entravent l'accès des femmes au marché du travail avec des postes et des salaires équivalents à ceux des hommes, affaiblissent encore plus capacité de celles-ci à s'émanciper de contextes de violence domestique. Sur la définition de la violence domestique, voir B. MEYERSFELD, Domestic Violence and International Law, Oxford, Hart Publishing, 2010. Voir également M. N. BERNARDES, "Domestic Violence and Gender Oppression : an Analysis of Brazilian Law in Light of a Theory of Democratic Justice", In M. ZIRK-SADOWSKI, B. WOJCIECHOWSKI, M. K. CERN (eds.), Towards the Recognition of Minority Groups : Legal and Communication Strategies, Londres, Ashgate Publishing Ltd, 2014.

15. Voir le site de l'ONG CFemea. Voir également IPEA, “Avaliando a efetividade...", op. cit., p. 10. 16. Voir W. PASINATO, "10 anos de Lei Maria da Penha. O que queremos comemorar ?", Revista Internacional de Direitos Humanos, vol. 1, 2017, p. 100

17. IPEA, "Avaliando a efetividade...", op. cit., p. 10.

18. L'étude de l'IPEA comparait le taux d'homicides commis au domicile sur des hommes, taux qui continuait d'augmenter, alors que le taux concernant les femmes restait stable. Le taux d'assassinat de femmes au domicile était de 1,1 pour 100000 habitants, en 2006, puis 1,2 pour 100 000, en 2011. Les morts violentes d'hommes chez eux sont passées de 4,5 pour 100 000, en 2006, à 4,8 pour 100 000, en 2011, IPEA, “Avaliando a efetividade...”, op. cit., p. 10.

19. Ver IPEA, “ Avaliando a efetividade...”, op. cit., p. 20.

20. Rapport final de le Commission parlementaire mixte d'enquête qui a étudié la violence contre la femme au Brésil et a enquêté sur des plaintes pour omission des pouvoirs publics à l'égard de l'application des dispositifs mis en place par la loi pour protéger les femmes victimes de violence, publié en juillet 2013.

21. Ce constat a alimenté la critique contre le prétendu caractère punitif de la LMP. Celle-ci serait au niveau d'autres manifestations d'un Etat pénal sélectif qui, indéniablement, opprime les populations subalternisées (pauvres et noirs). Je suis d'accord dès lors qu'il s'agit de dénoncer la capture de la loi par la logique pénale, mais je pense que vis-à-vis des lois conquises par les mouvements sociaux - comme c'est le cas de la loi Maria da Penha, de la loi contre le racisme et, si elle est votée, de la loi contre l'homophobie -, on ne doit pas faire d'amalgame. Comme le dirait Nancy Fraser, cela reviendrait à ajouter "l'insulte à l'offense": quand des populations d'habitude opprimées par l'État parviennent finalement à réorienter certains aspects du droit pénal en leur faveur, on finit par les traiter de punitivistes et de conservatrices! Carmen Hein Campos, membre du groupe de féministes qui a rédigé l'avant-projet de la LMP, parle d'un nécessaire virage paradigmatique dans l'interprétation de la loi, pour aller au-delà du droit pénal et explorer les autres potentialités qu'elle ouvre. Rodrigo Costa montre comment la loi, même dans l'interprétation restrictive et répressive qui en est faite, n'a augmenté la population carcérale que de façon très marginale : C. H. CAMPOS, "Lei Maria da Penha, a necessidade de um 
novo giro paradigmático", Revista Brasileira de Segurança Pública, São Paulo, vol. 11, n. 1, 10-22, JanFev. 2017.

22. Voir IPEA, « Avaliando a efetividade... », op. cit., p. 12.

23. Pour une critique progressiste sur cette innovation, voir A. L. P. FLAUZINA, «Lei Maria da Penha: entre os anseios da resistência e as posturas da militância », in . L. P. FLAUZINA, F. FREITAS, H. VIEIRA. T. PIRES (eds.), Discursos Negros : legislação penal, política criminal e racismo, Brasília, Brado Negro, 2015.

24. Sur l'impact de la LMP sur la population carcérale, voir R. de S. COSTA, A. V. OLIVEIRA, "Lei 11.340/06 e sistema penal : o quão punitivos são os juizados de violência doméstica e familiar contra a mulher", in E. J. TEIXEIRA J.L.A. BARBOSA, P. R. de LIMA FALCÃO, (eds.), Direitos, gênero $e$ movimentos sociais II, 1 éd. Florianópolis, CONPEDI, 2014, vol. 1, pp. 267-282.

25. A ce propos, voir M. FOUCAULT, História da Sexualidade - a vontade de saber, vol. 1. a ed, São Paulo, Graal, 2001. Judith Butler parle de la précarité comme condition qui définit l'existence : tous les êtres vivants sont vulnérables à la privation, aux offenses, aux lésions de l'intégrité physique et à la mort en vertu de dynamiques qu'ils ne maîtrisent pas. Nous sommes également et inexorablement dépendants d'interaction avec les services d'infrastructures sociales et institutionnelles pour avoir ce qu'elle appelle « une vie vivable». Toutefois, l'auteure souligne aussi que les conditions de la précarité ne sont pas également réparties sur le globe et certains corps sont plus exposés que d'autres à la privation et à la violence. Si on ne tient pas compte de cette répartition inégalitaire, à chaque fois que l'on mobilise un langage et des stratégies « inclusives » et universalisantes, on contribue aux processus de soumission, J. BUTLER, Notes toward a Performative Theory of Assembly. Cambridge, Harvard University Press, 2015, pp. 18-19. Cet argument est développé par l'auteure depuis Quadros de Guerra, publié en 2006, et Precarious Lifes, publié en 2009.

26. Sur la violence épistémique, le texte « Les subalternes peuvent-elles parler ? ", de G. SPIVAK, (op. cit.) fait référence. Spivak est l'une des cheffes de file de la pensée postcoloniale et des études subalternes, dont les dénonciations et les revendications sont proches, bien que différentes, de la pensée décoloniale.

27. M. LUGONES, "Rumo a um feminismo descolonial", Estudos Feministas 22(3), setembrodezembro 2014, p. 936.

28. M. LUGONES, op. cit., p. 938.

29. L. GONZALEZ, "Racismo e Sexismo na Cultura Brasileira", Revista Ciências Sociais Hoje, ANPOCS, 1984, p. 228. Pour un exemple classique de la critique du mythe de la fragilité féminine, voir l'ouvrage de Betty Friedan: B. FRIEDAN, The Feminine Mystique, New York, W.W. Norton \& Company, Inc. 1963.

30. S. CARNEIRO, «Enegrecer o feminismo : a situação da mulher negra na América Latina a partir da perspectiva de gênero. ", janvier 2019, pp. 1-2.

31. T. M. C. ALMEIDA, B. C. J. PEREIRA, "Violência Doméstica e Familiar contra mulheres pretas e pardas no Brasil : reflexão pela ótica dos estudos feministas latinoamericanos”, Critica e Sociedade : Revista de cultura política, vol. 2, n. 2, Dossiê : Cultura e Política, dez 2012, pp. 58-59.

32. B. J. PEREIRA, Tramas e dramas de gênero e de cor : a violência doméstica contra mulheres negras, Brasília, Brado Negro, 2016, p. 110.

33. B. J. PEREIRA, op.cit., p. 61.

34. B. J. PEREIRA, op.cit., p. 90. Pereira affirme que les femmes noires et mulâtresses subissent couramment des formes de discrimination raciale de la part de leurs compagnons et parents, qu'ils soient noirs ou non. Diverses enquêtées déclaraient que la violence psychologique teintée de racisme faisait plus mal que la violence physique : être traitée de "vagabunda ", que ce soit dans le sens d' « infidèle" ou de "fainéante », était ressenti comme particulièrement offensif, surtout lorsque l'agresseur le faisait en allusion au fait d'être noires. Compte tenu de la représentation sociale hyper sexualisée de la femme noire, il leur est encore plus exigé de 
prouver qu'elles ne sont pas infidèles. De la même manière, la représentation de la femme noire en tant que «travailleuse infatigable » modifiait aussi les attentes vis-à-vis du contrat sexuel du travail, PEREIRA B. J., op. cit., pp. 74, 134 et s.

35. K. CRENSHAW, "Mapping the Margins: Intersectionality, Identity Politics, and Violence against Women of Color", Stanford Law Review, vol. 43, n 6 (jul., 1991), p. 1241.

36. K. CRENSHAW, "Documento para encontro de especialistas em aspectos da discriminação racial relativos ao gênero", op. cit., pp. 5-6.

37. K. CRENSHAW, "Documento para encontro de especialistas em aspectos da discriminação racial relativos ao gênero ", op.cit., p. 5.

38. C'est Marília Montenegro qui a attiré mon attention sur les problèmes soulevés par la priorisation de la réponse judiciaire dans la lutte contre la violence domestique au Brésil.

39. A. L. P. FLAUZINA, op. cit., pp. 132.

40. K. CRENSHAW, "Mapping the Margins", op cit., p. 1259.

41. Ibid., pp. 1245-1246 et p. 1251.

42. M. N. BERNARDES, M. I. ALBUQUERQUE, op.cit, p. 729.

43. Ibid., p. 731.

44. Ibid., p. 718.

45. M.N. BERNARDES, R. S. COSTA, A. V. OLIVEIRA, op.cit., p. 134.

46. M. MONTENEGRO, Lei Maria da Penha : uma análise criminológico-crítica, Rio de Janeiro, Revan, 2015, pp.171-172. Il faut noter que Montenegro, ayant recours au cadre théorique de la criminologie critique - que, pour l'instant, je laisse de côté-, aboutit à des conclusions moins optimistes que moi quant aux potentialités émancipatrices de la LMP.

47. A. L. P. FLAUZINA, op. cit., p. 138.

48. Pour Flauzina, la résistance serait formée par ces femmes qui fraternisent comme « rescapées du quotidien ", alors que les militantes seraient la "rébellion politisée », A. L. P. FLAUZINA, op. cit., p. 116.

49. Après le LMP, il est possible de poursuivre et de condamner quelqu'un pour violence domestique. Avant le LMP, ces cas n'avaient jamais abouti à des condamnations. Ils avaient tous été résolus via des "transactions criminelles » qui tout au plus contraignait l'agresseur à payer des frais.

50. Citant les chiffres de l'étude de l'IPEA de 2012, l'auteure explique que les femmes victimes de violence domestique sont demandeuses d'écoute de leur expérience, de respect de leur douleur et d'arrêt de la violence. Elles ne cherchent pas l'incarcération de l'agresseur. Les combats des militantes pour interdire la possibilité de rétractation de la victime dans les procès pour lésion corporelle légère et la possibilité de suspension conditionnelle du procès, sont, d'après l'auteure, des choix qui posent problème parce qu'ils vont à l'encontre de ces demandes. A. L. P. FLAUZINA, op. cit., p. 129.

51. B. HOOKS, Feminist Theory : from margins to center, South End Press, 1984. p. 51.

\section{RÉSUMÉS}

Cet article analyse la façon dont le racisme structurel affecte la construction des hiérarchies de genre au Brésil, en se concentrant sur le problème de la violence domestique dans ce pays. En s'appuyant sur les analyses féministes décoloniales et noires, l'article examine comment le 
racisme a eu des effets sur les résultats de la loi « Maria da Penha », importante loi rédigée par des féministes et considéré comme un point d'accomplissement au Brésil. Pourtant, alors que le nombre de féminicides commis contre les femmes blanches au Brésil a diminué au cours de la dernière décennie, le nombre de féminicides commis contre les femmes noires a augmenté. En examinant les diverses stratégies des féministes " hégémoniques » contre la violence sexiste, l'article soutient que le racisme a produit une invisibilité des femmes noires comme actrices de leur propre émancipation.

This article discusses ways in which structural racism affects the construction of gender hierarchies in Brazil, focusing on the problem of domestic violence in the country. Resorting to decolonial and black feminist insights, it reflects on how racism impacts the results of the Maria da Penha Law, an important piece of legislation drafted by feminists, which is considered to be an important achievement in the contry. While the number of feminicides committed against white women in brazil has decreased in the past decade, the number of feminicides committed against black women has increased. Reviewing hegemonic feminist strategies against gender violence, it argues that racism has produced an invisibilty of black women as agents of their emancipation.

\section{INDEX}

Mots-clés : racisme, blanchitude, violence domestique

Keywords : racism, whiteness, domestic violence

\section{AUTEUR}

\section{MÁRCIA NINA BERNARDES}

Márcia Nina Bernardes est professeure de droit à la Pontifical Catholic University of Rio de Janeiro (PUC-Rio). Elle coordonne le centre "PUC-Rio Human Rights". Elle est membre de la LatinAmerican Scholar on Gender and Sexuality (RED Alas) : marcianb@puc-rio.br 